\title{
Natural language analysis to detect Parkinson's disease
}

\author{
P. A. Pérez-Toro ${ }^{1}$, J. C. Vásquez-Correa ${ }^{1,2}$, M. Strauss ${ }^{2}$, J. R. Orozco-Arroyave ${ }^{1,2}$, \\ and E. Nöth ${ }^{2}$ \\ ${ }^{1}$ Faculty of Engineering, University of Antioquia UdeA, Medellín, Colombia \\ \{paula.perezt, jcamilo.vasquez, rafael.orozco\} @udea.edu.co \\ 2 Pattern Recognition Lab, Friedrich-Alexander-Universität Erlangen-Nürnberg, Germany. \\ \{juan.vasquez, martin.straauss, elmar.noeth\}@fau.de
}

\begin{abstract}
Parkinson's disease (PD) is a neuro-degenerative disorder that produces motor and non-motor impairments. Non-motor impairments include communication and mood disorders. Most of the studies in the literature have been focused on the analysis of motor symptoms. However, non-motor signs are also present in most of the cases. This paper addresses the study of language production as a potential tool to diagnose and monitor the neurological state of PD patients. The study proposes the use of natural language processing methods to extract features from transcriptions obtained from spontaneous speech recordings to discriminate between healthy control people and PD patients. The analysis considered classical features such as Bag of Words and Term Frequency-Inverse Document Frequency, along with methods based on word-embeddings. Accuracies of up to $72 \%$ are obtained when discriminating between PD patients and healthy subjects, which confirms that there is information embedded in the language production that can be used for the assessment of the disease.
\end{abstract}

Keywords: Parkinson's disease, Natural language processing, Text processing, Language Analysis, Classification

\section{Introduction}

Parkinson's disease (PD) is a neuro-degenerative disorder characterized by the progressive loss of dopaminergic neurons in the mid brain [1]. Communication problems and impairments in the grammar production appear in $90 \%$ of the patients due to the death of the dopaminergic neurons [2]. The standard scale to evaluate the neurological state of the patients is the Movement Disorder Society-Unified Parkinson's Disease Rating Scale (MDS-UPDRS-III) [3], which includes items related to the speech production, mental state, and body movements, and others. The third section (MDS-UPDRS-III) of the scale considers motor activities of patients. It has a total of 33 items, which range from 0 to 4 , for a total range from 0 (completely healthy) to 142 (completely impaired). Most of the studies in the literature have been more focused on the speech analysis rather than on the language comprehension. Several studies suggest that, besides articulatory problems, impairments in grammar, verbal fluency, and semantic are also present in most of the patients $[4,5]$. There are few studies analyzing language impairments due to PD. In [6] the authors analyzed different components of text on transcribed monologues produced by $51 \mathrm{PD}$ patients and 50 healthy control $(\mathrm{HC})$ subjects. The analysis was performed using latent semantic analysis, part-of-speech tagging and word-level repetitions via graph embedding tools. The authors considered several classifiers to discriminate between PD patients and HC subjects, including a support vector machine 
(SVM) with a radial basis function (RBF) kernel, a K-nearest neighbors, and an Adaboost algorithm. The authors reported accuracies of up to $66 \%$ using latent semantic analysis, $75 \%$ using part-of-speech tagging and $77 \%$ using graph embedding tools.

Cognitive deficits and behavioral disorders are more common in Alzheimer's disease (AD) than in PD. Thus, besides the studies on PD, there are several works on AD where the impact of language impairments is studied [7-9]. This opens more possibilities of finding frameworks and methodologies that could be adapted to address the problem of language modeling in PD. Recently in [9] the authors proposed an approach of counting word occurrences in transcriptions via Bag of Words (BoW) vectors. English transcriptions from the Pitt Corpus of the Dementia Bank [10] (168 AD patients and 94 $\mathrm{HC}$ subjects), were considered. The participants were asked to describe the cookie theft image [11]. BoW features were used to classify the AD patients and $\mathrm{HC}$ subjects using an artificial neural network. The authors followed a Leave-One-Speaker-Out (LOSO) cross-validation strategy, and reported accuracies of up to $84.4 \%$.

Although acoustic analysis has shown to be a suitable tool to study symptoms of PD patients, there are components related to language production that are not modeled with that approach. With the aim to model language deficits exhibited by PD patients, this study considers the use of several Natural Language Processing (NLP) methods including classical approaches such as BoW and Term Frequency-Inverse Document Frequency (TF-IDF), along with novel methods based on word-embeddings like word2vec (W2V). Experiments with recordings of spontaneous speech produced by 50 PD patients and 50 healthy subjects are considered. Accuracies of up to $72 \%$ are obtained, which confirms the results reported in similar studies.

\section{Data}

Recordings of spontaneous speech of $50 \mathrm{PD}$ patients and $50 \mathrm{HC}$ subjects are manually transcribed. The speech recordings are part of the PC-GITA corpus [12]. For this paper only recordings of monologues are considered. The task consisted on asking the participants to talk about their daily routines. The average duration of the monologues is $48 \pm 29$ seconds for the patients and $45 \pm 24$ for the healthy subjects. The transcriptions were produced following the verbatim protocol, using headphones to maximize the transcription accuracy and to minimize possible human errors. The whole vocabulary of the transcriptions without stopwords (e.g., the, of, in, on, etc.) consists of 1182 words. The patients were evaluated by an expert neurologist and labeled according to the MDS-UPDRS-III score. Table 1 shows additional information of the subjects.

Table 1: General information of the subjects. Time since diagnosis, age and education are given in years. ${ }^{a} p$ calculated through Chi-square test. ${ }^{b} p$ calculated through $\mathrm{t}$ test.

\begin{tabular}{lccc}
\hline & PD patients & Healthy controls & Patients vs. controls \\
\hline Gender [F/M] & $25 / 25$ & $25 / 25$ & $p=1.00^{a}$ \\
Age [F/M] & $60.7(7.3) / 61.3(11.7)$ & $61.4(7.1) / 60.5(11.6)$ & $p=0.98^{b}$ \\
Education [F/M] & $11.5(4.1) / 10.9(4.5)$ & $11.5(5.2) / 10.6(4.4)$ & $p=0.88^{b}$ \\
Time since diagnosis [F/M] & $12.6(11.5) / 8.7(5.8)$ & & \\
MDS-UPDRS-III [F/M] & $37.6(14.0) / 37.8(22.1)$ & & \\
\hline \hline
\end{tabular}




\section{Methods}

NLP approaches are considered to process information from the texts resulting from the transcription process. Figure 1 shows the main steps followed in this study to perform the text analysis. Details of the processes performed on each stage are included in the following subsections.

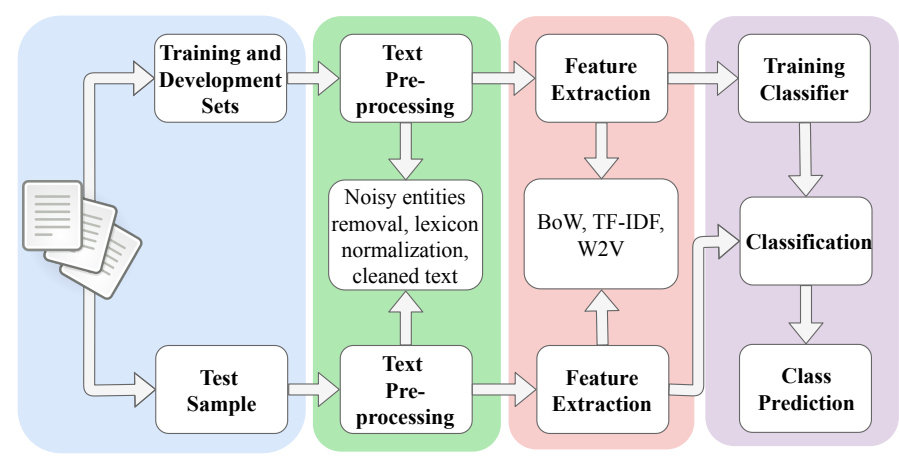

Fig. 1: Scheme of the general methodology addressed in this study.

\subsection{Train, development, and test data distribution}

The data is distributed following a LOSO strategy as it is shown in Figure 2. There is always a test subject and the remaining data is used for the process of training and development which is performed following a 10-fold cross validation strategy to optimize the meta-parameters of the classifier. The optimization criterion is the accuracy obtained in development. This process can be seen as a set of independent experiments over the training data. We are aware about the fact that this process might be slightly optimistic since we have a different set of meta-parameters for each test sample; however, the results show that the distribution of the meta-parameters is stable across the different test sets.

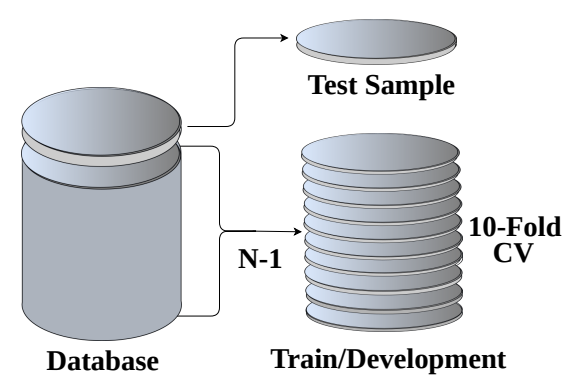

Fig. 2: Database distribution. CV: cross-validation. N: number of samples. 


\subsection{Pre-Processing}

The data is cleaned and standardized, making it noise-free and ready for analysis as it is shown in Figure 1. The following two steps are implemented for this purpose:

- Noise removal: non relevant information for the context, such as stopwords, accents or punctuation, are removed from the text. We considered a Spanish dictionary of noise entities from the Natural Language Toolkit (NLTK) [13] to remove the noise words from the transcriptions.

- Lexicon normalization: there are multiple representation for a single word. To standardize the words in an equal representation, all words were transformed via stemming to remove the suffixes. Another implemented method was lemmatization, which transforms the words into their root form.

\subsection{Feature extraction}

Bag of Words-BoW

This method creates a vocabulary of all words that appear in the whole document, i.e., it is a collection of words that will be represented into a feature vector with fixed size. The process to extract the BoW feature vector includes the following steps: (1) the sentences are represented as a collection of words. (2) Each sentence is pre-processed and tokenized. (3) The vocabulary is created, and multiple occurrences of the same word are removed. (4) The words of the entire corpus are counted and stored in a vector with a length of 1182 words (the total number of words in the corpus). A more realistic feature set could be formed only with the words from the training set, by considering the words from the test set that are not in the vocabulary as out-of-vocabulary words.

\section{Term Frequency-Inverse Document Frequency}

Term frequency (TF) gives the relative frequency of a specific term, word or combination of words. This value is compared to the relative frequency of other terms in a text or document. Inverse Document Frequency (IDF) compares the number of all available documents and the number of documents that contain the target word. IDF determines the relevance of the text with respect to a specific word. Finally, TF-IDF features are expressed by Equation 1 for the word $W_{i, j} . \mathrm{TF}_{i, j}$ is the number of occurrences of the term $i$ in the document $j, d_{f_{i}}$ is the number of documents containing $i$, and $N$ is the total number of documents.

$$
W_{i, j}=\mathrm{TF}_{i, j} \log \left(\frac{N}{d_{f_{i}}}\right)
$$

\section{Word2 Vec-W2V}

The aim of $\mathrm{W} 2 \mathrm{~V}$ is to represent the words as a vector in a multidimensional space, where similar or related words are represented by nearby points. The model consists of a Neural Network (NN) with one hidden layer. The activations of the hidden layer are stored, being those "the word vectors". The input words are represented as one-hot encodings, i.e., as binary vectors. Neighbor words in this representation are considered to train the NN. The selection of the neighbor words depends on the "window size", which is necessary to model the temporal context of each word. 
For our experiments, the NN was trained using the Spanish WikiCorpus, which contains 120 million of words [14]. The window size was fixed in 7, because it was the average number of words per sentence in the transcripts. The length for the word-embedding vector was defined in 100. Then, four statistical functionals were computed: mean, standard deviation, skewness, and kurtosis, forming a 400dimensional feature vector per transcription.

\subsection{Classification}

Kruskal-Wallis tests were performed to assess whether there is a significant difference between the median of the features computed for PD patients and HC subjects. The null hypothesis of the medians coming from the same distribution was rejected $(p \ll 0.05)$ in all cases. The discrimination capability of the extracted features is evaluated using two classifiers, RBF-SVM and Random Forest (RF). The criterion for the meta-parameters optimization is based on the accuracy obtained in the development data. Optimal parameters are found through a grid search where $C \in\left\{10^{-4}, 10^{-3}, \ldots 10^{4}\right\}$ and $\gamma \in\left\{10^{-4}, 10^{-3}, \ldots 10^{4}\right\}$ for the RBF-SVM, and number of trees $N \in\{5,10,20,30,50,100\}$ and $D \in\{2,5,10,20,30,50,100\}$ for the RF. The performance of the classifiers is evaluated considering the accuracy, sensitivity, specificity, and the area under the receiving operating characteristic curve (AUC).

\section{Results and discussion}

In order to visualize linguistic differences between PD and HC subjects, Figure 3 shows a word cloud representation Note words as "casa" (house) or "ver televisión" (watch television) are more frequently used for the patients, while words as "trabajar" (work) or "salir" (go out) appear more frequently in HC subjects. In addition to the words related to the daily activities of the subjects, in PD patient appears more frequently a Colombian crutch "pues", denoting a lack of fluency in the speech of the patients.

A

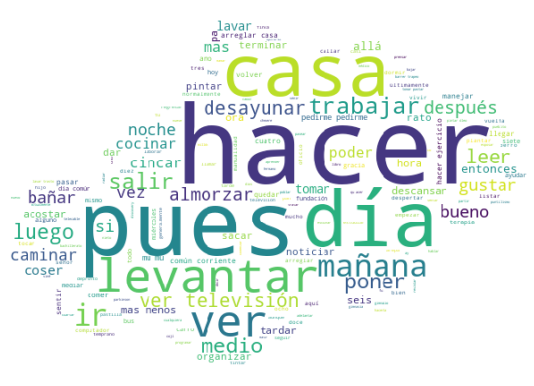

B

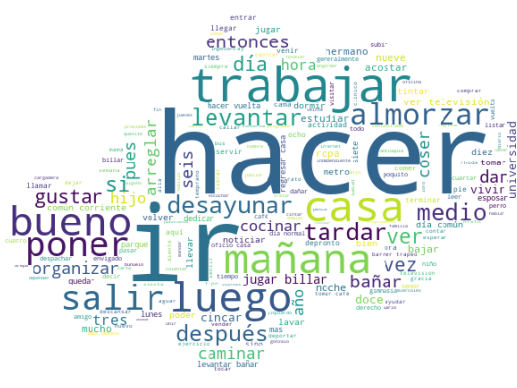

Fig. 3: Word cloud representation: A) PD patient. B) HC subject.

Results considering the three feature sets individually and their combination in an early fusion strategy are shown in Table 2. Note that in general sensitivity is higher 
than specificity, which indicates that PD patients were better discriminated in most of the cases. This difference between specificity and sensitivity suggests that AUC is a better statistic to compare the approaches. The highest AUC is observed with the BoW features classified with the RF (0.76). Note also that the fusion strategy did not improve the results indicating that the considered features are not complementary and further research is required to find an optimal strategy to merge such information.

Table 2: Classification results.

\begin{tabular}{l|cccccc|cccccc}
\hline Features & \multicolumn{9}{|c|}{ RBF-SVM } & \multicolumn{8}{c}{ RF } \\
& Acc(\%) & Sens(\%) & Spe(\%) & AUC & C & $\gamma$ & Acc(\%) & Sens(\%) & Spe(\%) & AUC & N & D \\
\hline BoW & 62.0 & 70.0 & 54.0 & 0.60 & $10^{10}$ & $10^{0}$ & 70.0 & 74.0 & 66.0 & 0.76 & 100 & 20 \\
TF-IDF & 58.0 & 58.0 & 56.0 & 0.60 & $10^{1}$ & $10^{0}$ & 67.0 & 68.0 & 66.0 & 0.71 & 100 & 100 \\
W2V & 72.0 & 92.0 & 52.0 & 0.66 & $10^{0}$ & $10^{0}$ & 67.0 & 74.0 & 60.0 & 0.71 & 5 & 5 \\
Fusion & 60.0 & 62.0 & 58.0 & 0.62 & $10^{1}$ & $10^{0}$ & 66.0 & 68.0 & 64.0 & 0.71 & 100 & 5 \\
\hline \hline
\end{tabular}

Notes: Acc: accuracy. Sens: sensitivity. Spe: specificity. AUC: Area under the ROC curve. C and $\gamma$ are the optimal meta-parameters obtained in development for RBF-SVM. $\mathbf{N}$ and $\mathbf{D}$ are the optimal meta-parameters obtained in development for RF.

With the aim to compare the approaches that provided better AUC values, the distribution of the scores obtained with the RF classifier for the two classes considering the BoW and TF-IDF features are shown in Figures 4.A and 4.B, respectively. The dark gray bars correspond to the scores for $\mathrm{HC}$ subjects, the white bars are the scores computed for the PD patients, and the light gray bars correspond to the intersection between both sets, and reflect the classification errors. Note that the scores of the PD patients are less sparse than those obtained for the HC subjects, additionally the scores for the TF-IDF features are more overlapped. Figure 5.A and Figure 5.B show the score distributions obtained also with the RF classifier when considering W2V features and the fusion, respectively. Note that these scores are more overlapped and sparse, especially for $\mathrm{HC}$ subjects, were a higher variance is observed.

The results found here are similar to others reported in the state-of-the-art, and show to be complementary to those reported previously, where we performed an acoustic analysis of the daily routine monologues performed by the patients [15]. We believe that they could improve if the participants were evaluated in conversational speech samples or in recordings while describing a scene or a story (retelling tasks). Further research with these kinds of experiments is required to find more discriminant approaches.

\section{Conclusion}

Several NLP techniques were considered in this paper to discriminate between HC subjects and PD patients. The feature sets included classical methods such as BoW and TF-IDF along with other techniques based on word-embeddings like W2V. The main aim was to analyze the suitability of NLP methods to discriminate PD vs. HC subjects. The proposed approach allows the study of different communication disorders that cannot be observed in motor activities. Thus this is a step forward in the evaluation of language impairments that affect the communication capabilities of PD patients. The proposed models are relatively accurate to classify PD patients and HC subjects. As 

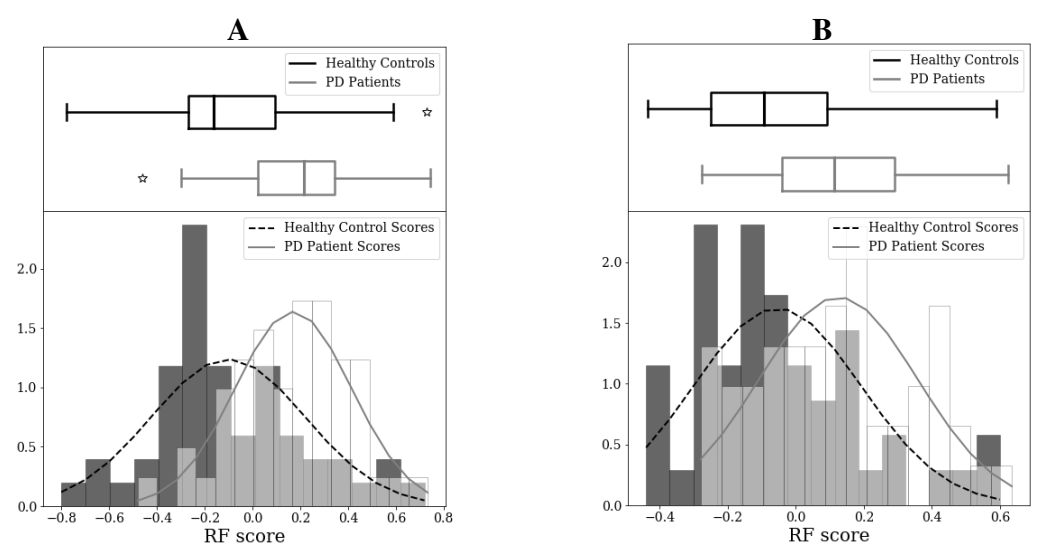

Fig. 4: Scores obtained for the RF classifier for: A) BoW. B) TF-IDF.
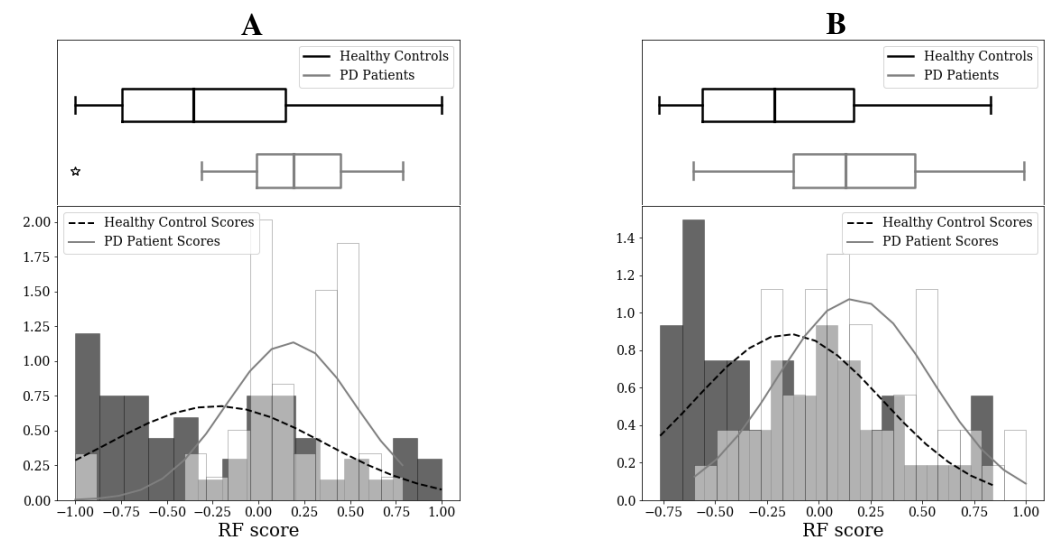

Fig. 5: Scores obtained for the RF classifier for: A) W2V. B) Fusion.

it was also observed in previous studies, the results obtained here suggest that there is information that reflects language impairments in PD patients and it can be used to discriminate between PD and $\mathrm{HC}$ subjects and also to evaluate the neurological state of the patients.

Although we think that the results reported here are a step towards the automatic evaluation of language impairments in PD patients, the main limitation is related to the restricted task performed by the participants. They were asked to describe their daily routines. According to our observations, it seems like this task does not reflect communication deficits of PD patients properly because there is an implicit bias in the recorded task. Healthy subjects tend to do more active activities than PD patients who do mainly passive activities like reading, thinking, and taking their medication. Our team is currently collecting more recordings with the aim of evaluating the suitability of other tasks like image description. Further experiments will explore more robust word embedding methods such as ELMo or BERT to improve the performance of the system. 
Additionally, we are also working on the fusion of acoustic and language information to see whether the results can be improved.

\section{References}

1. O. Hornykiewicz. Biochemical aspects of Parkinson's disease. Neurology, 51(2 Suppl 2):S2S9, 1998.

2. K.M. Smith and D.N. Caplan. Communication impairment in Parkinson's disease: Impact of motor and cognitive symptoms on speech and language. Brain and Language, 185:38-46, 2018.

3. C.G. Goetz et al. Movement Disorder Society-sponsored revision of the Unified Parkinson's Disease Rating Scale (MDS-UPDRS): Scale presentation and clinimetric testing results. Movement Disorders, 23(15):2129-2170, 2008.

4. L.L. Murray and L.P. Lenz. Productive syntax abilities in Huntington's and Parkinson's diseases. Brain and Cognition, 46(1-2):213-219, 2001.

5. S. Vanhoutte, M. De Letter, P. Corthals, et al. Quantitative analysis of language production in Parkinson's disease using a cued sentence generation task. Clinical linguistics \& phonetics, 26(10):863-881, 2012.

6. A.M. García, F. Carrillo, J.R. Orozco-Arroyave, N. Trujillo, et al. How language flows when movements don't: an automated analysis of spontaneous discourse in Parkinson's disease. Brain and Language, 162:19-28, 2016.

7. K.C. Fraser, J.A. Meltzer, and F. Rudzicz. Linguistic features identify Alzheimer's disease in narrative speech. Journal of Alzheimer's Disease, 49(2):407-422, 2016.

8. S. Wankerl, E. Nöth, and S. Evert. An N-gram based approach to the automatic diagnosis of Alzheimer's disease from spoken language. In Proceedings of the Annual Conference of the International Speech Communication Association, INTERSPEECH, 2017.

9. P. Klumpp, J. Fritsch, and E. Noeth. ANN-based Alzheimer's disease classification from bag of words. In Speech Communication; 13th ITG-Symposium, pages 1-4. VDE, 2018.

10. J. T. Becker et al. The natural history of Alzheimer's disease: description of study cohort and accuracy of diagnosis. Archives of Neurology, 51(6):585-594, 1994.

11. H. Goodglass and E. Kaplan. Boston Naming Test: scoring booklet. Lea \& Febiger, 1983.

12. J.R. Orozco-Arroyave, J.D. Arias-Londoño, J.F. Vargas-Bonilla, et al. New Spanish speech corpus database for the analysis of people suffering from Parkinson's disease. In $L R E C$, pages 342-347, 2014.

13. S. Bird and E. Loper. NLTK: the natural language toolkit. In Proceedings of the ACL 2004 on Interactive poster and demonstration sessions, page 31. Association for Computational Linguistics, 2004.

14. S. Reese, G. Boleda, M. Cuadros, et al. Wikicorpus: A word-sense disambiguated multilingual wikipedia corpus. 2010.

15. J. C. Vásquez-Correa, J. R. Orozco-Arroyave, and E. Nöth. Convolutional neural network to model articulation impairments in patients with Parkinson's disease. In Proceedings of INTERSPEECH, pages 314-318, 2017. 